in solids. So far as the growth of explosion is concerned we need more experimental results. In particular, there is even a lack of data of quantities such as thermal conductivity and specific heat. The disintegration and break-up of solids ahead of the reaction zone have been stressed by a number of workers. The formation of a molten zone and its importance, both in the growth of explosion and in low-velocity detonation, have been mentioned. With the development of new apparatus, and with the techniques now available, we may expect a rapid advance in the near future on this problem of the growth of reaction to detonation.

\section{A. D. YoFfe}

\section{RECENT ADVANCES IN HIGH POLYMER PHYSICS}

A

CONFERENCE on "Rheology of Elastomers" was held by the British Society of Rheology during May 29-31 at the British Rubber Producers' Research Association, Welwyn Garden City.

Equilibrium properties of rubberlike materials. In an introductory talk, Dr. L. R. G. Treloar (British Rayon Research Association) said that in the main the properties of rubber and of other polymeric materials in the highly elastic or 'rubberlike' state are now well understood. The stress and birefringence of rubber subjected to different types of finite strain at various temperatures are described to a good irst approximation by the 'kinetic theory', a relatively simple molecular theory which treats of the changes of configurational entropy and molecular orientation in a deforming network composed of flexible long-chain molecules. Reviewing the shortcomings of the theory (in the Gaussian approximation), Dr. Treloar said that for simple elongations in the range 150-500 per cent, the discrepancies between the theoretical and observed stress-strain relations have so far not received satisfactory 'molecular' explanation.

A crucial test of the applicability of the kinetic theory to polymeric materials is furnished by the prediction that the rigidity modulus should increase linearly with the absolute temperature and with the concentration of junctions in the network. By using gelatin gels specially prepared to give junctions which are substantially unaffected by temperature changes, P. R. Saunders and A. G. Ward (British Gelatine and Glue Research Association) found that the rigidity modulus did increase linearly with temperature in the range $0^{\circ}-60^{\circ} \mathrm{C}$. and at a rate com. parable with that required by the theory. Data both from gel rigidities and from dilute solution viscosities at different $p \mathbf{H}$ indicated that differences in the rigidities of ordinary gelatin gels must be attributed to differences in concentration and strength of network junctions, rather than to differences in molecular chain 'stiffness'.

Improved techniques for conditioning filaments of natural rubber so as to yield a more accurate attain. ment of thermodynamic equilibrium (required for comparison with the kinetic theory) were described by $\mathbf{P}$. Thirion (Institut Français du Caoutchouc). For filaments held at constant extension in the range 0-30 per cent, reversibility of tension-temperature cycles $\left(20^{\circ}-60^{\circ}\right.$ C.) was attained to within 0.05 per cent. A new derivation was given of Gee's formula for determining the coefficients of change of entropy and internal energy with change of extension at constant volume and temperature. From the data, the corresponding coefficients at constant pressure and temperature were determined with accuracies of the order $0.03 \mathrm{gm} . / \mathrm{cm} .{ }^{2} / \mathrm{deg}$. C. and $10 \mathrm{gm} . / \mathrm{cm} .{ }^{2}$, respectively.

When the individual 'links' of long-chain molecules in a network have anisotropic optical polarizability, the network becomes birefringent when stressed. According to the kinetic theory, which assumes that no crystallization is present, the stress (for simple elongation) should be proportional to the magnitude of birefringence, and the constant of proportionality, or 'stress-optical coefficient', should not depend on the concentration of network junctions. Dr. D. W. Saunders (British Rayon Research Association) presented data obtained at temperatures above the crystal melting points which agreed with both these theoretical predictions in the case of cross-linked natural rubber and gutta percha. For polythene and polymethylene, however, the stress-optical coefficient decreased with increase of junction concentration; this was satisfactorily accounted for by extending the theory to apply to short-chain networks, and the data indicated that these two polymers had comparatively stiff chains. The problem of giving a strict definition of chain stiffness was considered.

'The creation and loss of junctions in a network deformed from its unstressed state were considered in a theoretical paper by Dr. J. Scanlan (British Rubber Producers' Research Association). Tobolsky and co-workers have postulated a form of strainenergy function which would be obtained if the actual network behaved as if it were two independent net. works, each of which obeyed the kinetic theory. Dr. Scanlan showed that, in the case in which junctions are created but none is lost, this postulate can be justified, but in the case in which one junction is lost for every one created (in the deformed state), the postulated strain-energy function is correct in form but contains coefficients which must be corrected. Two arguments were presented-one based on the network theory of James and Guth, and the other on a corrected form of a theory given by Scott and Stein.

Elastic recovery, flow and stress relaxation. A striking demonstration of high elastic recovery in a liquid was given by Dr. A. S. Lodge (British Rayon Research Association), who poured a liquid (containing about 5 per cent aluminium laurate in paraffin) out of a bottle; on sharply cutting the liquid stream with scissors, the upper part of the stream snapped back into the bottle. Dr. Lodge has applied the network theory of James and Guth and the theory of relaxing networks of Green and Tobolsky to the problem of elastic recovery in solutions of long. chain molecules; for the instantaneous recovery following steady laminar shear flow, the latter theory predicts an unexpected expansion in directions normal to the previous flow lines. The suggestion that this might contribute to the diameter increase commonly observed when polymer solutions and melts are extruded from tubes of circular crosssection gave rise to considerable discussion, and alternative explanations, also in terms of elastic recovery, were mentioned by Dr. P. Clegg (Imperial Chemical Industries, Plastics Division). Dr. Clegg's data for molten polythene showed that at low flow 
rates the diameter increase did not depend on tube length (in agreement with Dr. Lodge's theory) but did depend on tube length at higher flow rates when the transit time through the tube became comparable with the stress relaxation periods of the melt. In this case, the melt was presumably 'remembering' its convergent flow at the entry to the tube. The main part of Dr. Clegg's paper dealt with still higher flow rates, when marked irregularity occurred in the extruded filament. By observation of the motion of embedded particles and of coloured parts of the polymer, Dr. Clegg established that the cause of the irregularity lay in the conditions of flow in the region of entry to the tube ; a motion film was shown which clearly demonstrated very marked disturbances of flow in this region. Independent measurements of melt elastic properties suggested that polymers for which the irregular flow sets in at lower shear stresses (or rates) have longer stress relaxation times.

Unoriented polymers crystallize if left long enough at suitable temperatures; crystallization can be accelerated by stretching processes which cause appreciable molecular orientation. To separate these causes of crystallization as far as possible, Dr. E. L. Foster and H. Heap (Imperial Chemical Industries, Fibres Division) have constructed an apparatus with which a filament can be heated rapidly (in about 1 sec.) and its tension-extension characteristics measured. Data for amorphous polyethylene terephthalate ('Terylene') in the range $90^{\circ}-120^{\circ} \mathrm{C}$. showed that at low rates of strain the filament can be extended almost indefinitely with little increase in tension; at high rates of strain, however, the tension increases catastrophically with marked increase in crystallinity, as indicated by X-ray data.

Many measurements have been made of the relaxation of tension in a polymer strip which is rapidly extended and then maintained at constant extension. Prof. G. Gee, Or. G. Allen and B. E. Read (University of Manchester) have constructed an apparatus in which the electronic recording of tension enables measurements to be made during the first ten seconds when most of the relaxation occurs, as well as at later times. Data were presented for natural rubber at moderate extensions (up to 50 per cent) and at temperatures in the range $45^{\circ}-60^{\circ} \mathrm{C}$., so that erystallization would be very limited. The data taken at the longer time intervals were used to calculate values for the activation energy and activation flow volume.

Elastic recovery in polythene rods has been investigated by J. J. Benbow (Imperial Chemical Industries, Akers Research Laboratory). A rod was held twisted for a given time, released, and the subsequent untwisting measured as a function of time. Only at very small strains (about 0.1 per cent) did the results agree with a theoretical relation obtained by combining Boltzmann's theory of the 'elastic after-effect' with the empirical result that internal friction in polythene subjected to oscillatory strains is independent of frequency. Polythene appeared less able to 'remember' the duration of the imposed twist as the magnitude of this twist was increased. The temperature was controlled at $30^{\circ} \mathrm{C}$.; the maximum strain imposed was about 100 per cent.

Miscellaneous properties of polymeric materials. In the mechanical response of polymeric materials to oseillatory strains of very small amplitude, an increase of temperature can, in a sense, be compensated for by a decrease of frequency. Ferry has given a prescription for the appropriate changes of temperature and frequency scales whereby the data for dynamic modulus and dynamic viscosity for a given polymer can be superposed in master curves over a remarkably wide range. A. R. Payne (Research Association of British Rubber Manufacturers) gave a wealth of data showing the successful application of Ferry's method to various cross-linked and uncrosslinked rubbers, for dielectrical as well as for mechanical measurements. In these correlations, there is only one disposable constant, a characteristic temperature the value of which is assigned empirically for each polymer. Mr. Payne also discussed data already published for a wide variety of polymers with particular reference to the temperature variation of the frequency at which the maximum value of $\tan \delta$ occurs, where $\delta$ denotes the dielectric loss angle.

In order to obtain a rational measure of tear resistance of a cross-linked rubber, the growth of tears in rubber has been studied under controlled conditions. The concept and determination of a characteristic tearing energy was discussed by Dr. H. W. Greensmith (British Rubber Producers' Research Association). Measured values of this tearing energy showed much greater variation both with temperature and with rate of tearing for a synthetic rubber $(G R-S)$ than for a natural muber. The difference was attributed to the reinforcing effect of crystallization in the natural rubber. Addition of a filler to the synthetic rubber introduced a new maximum in the tearing energy when expressed as a function of temperature and tear rate.

Exposure of a polymer to radiation from an atomic pile or from a high-energy electron accelerator usually either creates cross-links (network junctions), or breaks chains, but rarely does both together. Mechanical properties of polymers are in the main the same, whether the cross-linking is effected by irradiation or by more conventional chemical means. Dr. A. Charlesby, Miss J. Burrows and T. Bain (Tube Investments Research Laboratories) have measured the modulus, stress-strain curve, elongation at break, and tensile strength for irradiated rubbers reinforced with carbon black, with sulphur, and with other additives. No significant difference was observed as between 'electron' radiation and pile radiation; the changes produced depended only on the total energy absorbed. There was some indication that chemical cross-linking gave a higher tensile strength than irradiative cross-linking. E. Fukada (Imperial College of Science and Technology, London) used $\gamma$-radiation to cross-link samples of a commercial polyester resin to which different amounts of styrene had been added. The dynamic modulus and mechanical damping at temperatures in the range $10^{\circ}-160^{\circ} \mathrm{C}$. at a single frequency of 0.22 cycle/sec. were obtained from observations of the horizontal and vertical deflexions of one end of a rod of the polymer which was rotated about its axis (held horizontally) by means of a rotating chuck at the other end.

The conference was attended by more than one hundred people, including visitors from Japan, Germany, France, and a substantial number from Holland. Discussions were always lively, and one was left with an impression of a flourishing field of investigation of considerable fundamental as well as technical interest. Publication of the papers and discussions is in the hands of Pergamon Press.

A. S. LODGE 